Check for updates

Cite this: RSC Adv., 2021, 11, 28761

Received 20th July 2021

Accepted 15th August 2021

DOI: 10.1039/d1ra05548h

rsc.li/rsc-advances

\section{Traditional uses, phytochemistry, pharmacology, and toxicology of Pterocephalus hookeri (C. B. Clarke) Höeck: a review}

\author{
Zhiqiang Gan, (D) $\dagger^{\mathrm{a}}$ Juan Jiang, $\dagger^{\mathrm{b}}$ Honglin Tao, ${ }^{a}$ Shiying Luo, ${ }^{a}$ Xianli Meng, ${ }^{\mathrm{c}} \mathrm{Jia} \mathrm{Yu},{ }^{* d}$ \\ Yi Zhang ${ }^{\star d}$ and Ce Tang*d
}

Pterocephalus hookeri (C. B. Clarke) Höeck is a member of the Dipsacaceae family and has been used in traditional Tibetan medicine for thousands of years. P. hookeri clears heat, detoxifies, stops dysentery, eliminates distemper, dispels wind, and relieves stagnation and is mainly prescribed for heat syndrome, dysentery, arthritis, and plague. Approximately 93 chemical compounds have been isolated and identified from $P$. hookeri, including iridoid glycosides, lignan and triterpenoids. Meanwhile, modern pharmacological studies have shown that $P$. hookeri has anti-inflammatory, anti-rheumatoid arthritis, analgesic, anticancer, and neuroprotection activities. However, studies on the in vivo pharmacokinetics and mechanism of action, discovery of quality markers, and qualitative and quantitative analysis are still insufficient. Hence, this paper provides a comprehensive review of the ethnic medicine, phytochemistry, pharmacology, and toxicology of $P$. hookeri to increase the understanding of the medicinal value of $P$. hookeri.
${ }^{a}$ School of Pharmacy, Chengdu University of Traditional Chinese Medicine, Chengdu 611137, China

${ }^{b}$ Chongqing University Cancer Hospital, Chongqing 400030, China

'Innovative Institute of Chinese Medicine and Pharmacy, Chengdu University of Traditional Chinese Medicine, Chengdu 611137, China

${ }^{d}$ School of Ethnic Medicine, Chengdu University of Traditional Chinese Medicine, Chengdu 611137, China. E-mail: 13515094@qq.com; zhangyi_mzyy@163.com; 409014182@qq.com

$\uparrow$ These authors contributed equally to this work.

\section{Introduction}

Pterocephalus hookeri (C. B. Clarke) Höeck is an herbaceous plant that belongs to the subfamily Pterocephalus in the Dipsacaceae family. The genus Pterocephalus currently includes 25 species which are mainly distributed in Europe, Asia, and Africa. Two species, P. hookeri and Pterocephalus bretschneideri (Batalin) E. Pritz. ex Diels, are found in China. P. hookeri is widely distributed in Sichuan, Yunnan, and Tibet (China) ${ }^{1,2}$ and is one of the most popular Traditional Tibetan Medicines, known as "푇하.

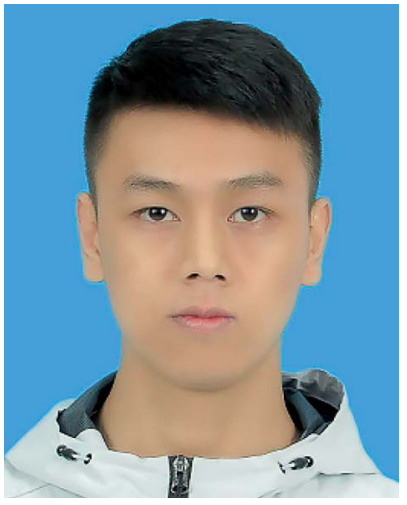

Zhiqiang Gan, male, studied at the School of Pharmacy, Chengdu University of TCM. From 2019 to present, he has been studying for a Master's degree in the chemistry of traditional Chinese medicine, under the tutelage of Dr Zhang $Y i$, the director of The Academic Inheritance and Innovation Research Center of Ethnic Medicine of Chengdu University of TCM. The research direction is the effective material basis of Chinese medicine and ethnic medicine.

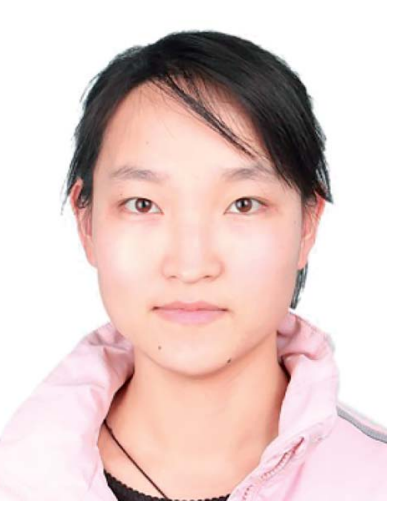

Juan Jiang, M. Sc, attending doctor. She graduated from Chongqing Medical University with a Master's degree in 2014. Since then, she has been working in the Chongqing University Cancer Hospital. She is currently an attending doctor in the Department of Traditional Chinese Medicine and has published many papers in Chinese journals. Her research direction is acupuncture. 
many classic Tibetan medicine books, such as The Four Medical Tantras (late 8th century AD) ${ }^{3}$ and Jing Zhu Materia Medica (19th century $\mathrm{AD}) .{ }^{4}$ The formal Chinese name of $P$. hookeri is Yishou-cao (Chinese name: 翼首草), which was first adopted by the Chinese Pharmacopoeia in $1977 . .^{5}$ P. hookeri is also known as Bang-zi-du-wu (Tibetan name: 쵣 के medicine and is widely used to treat cold, pain, plagues, and arthritis. Since 1993, the phytochemical and pharmacological research of $P$. hookeri has attracted widespread attention. ${ }^{6}$ To date, compounds isolated from $P$. hookeri, include: iridoids, triterpenoids, and phenylpropanoids. Modern pharmacological studies have demonstrated that $P$. hookeri possesses antiinflammatory, anti-rheumatoid arthritis, analgesic, antitumor, immunomodulatory, neuroprotection, and antibacterial activities.

This review aims to summarize comprehensive information on the botanical characteristics, distribution, traditional use, ethnopharmacology, chemical composition, pharmacological activity, and toxicity characteristics of $P$. hookeri, referencing ancient books and modern documents, to lay the foundation for further research on the mechanism of action of this traditional

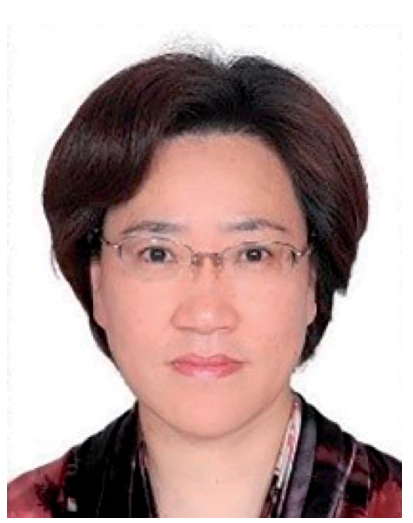

Xianli Meng, PhD, Professor, is the Dean of the Innovative Institute of Chinese Medicine and Pharmacy, Chengdu University of TCM. Professor Meng is the academic and technical leader of Sichuan Province and the review expert of National Natural Science Foundation of China evaluation. She has published more than 50 research papers in various SCI journals. As the leader of the team, Dr Meng is committed to the pharmacodynamics of traditional Chinese medicine and the application of NIR-II in the diagnosis of diseases.

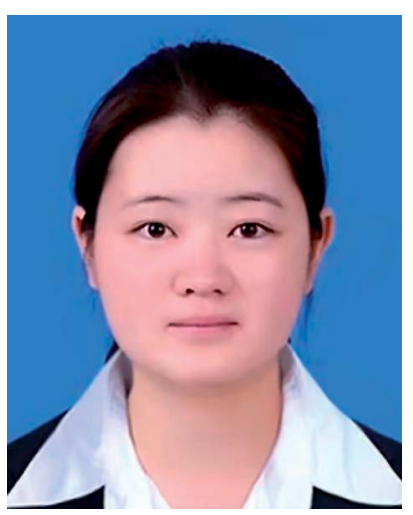

Jia Yu, lecturer, currently working at the School of Ethnic Medicine, Chengdu University of TCM. From 2019 to present, studying for a PhD, degree under the guidance of Dr Zhang Yi of Chengdu University of TCM. She has undertaken a number of scientific research projects and published more than 10 research papers in Chinese journals, and attended international and domestic conferences. Her current research direction is ethnic medicine and data informatization. medicinal plant and to guide the development of therapeutic drugs.

\section{Botanical characterization and distribution}

$P$. hookeri is a perennial herb with a height of $5-35 \mathrm{~cm}$. Its roots are conical, thick, and fleshy, and the upper part of the root is densely covered with brown residual petioles. Their leaves are basal, spatulate to oblong-oblanceolate, 3-20 cm long, and 1$4 \mathrm{~cm}$ wide, have curved coarsely dentate or pinnately lobed tips, and are tapered into stalks at the base, and have conspicuous midribs. Both sides of their leaves have thick and short villi (hairs). The scape is single, rarely two or three, and densely shirred. The capitulum is spherical, with a diameter of $2-3 \mathrm{~cm}$. The flowers are white or pink, and the bracts are like involucres, narrow. The epicalyx is tube-shaped, approximately $5 \mathrm{~mm}$ long, and pilose. The calyx is completely split into a pinnate crest. The corolla is funnel shaped, and $8-12 \mathrm{~mm}$ long. The apex has four or five lobes, and the crown tube is pubescent (fine short hairs) inside and outside. The stamens, usually four in number, are

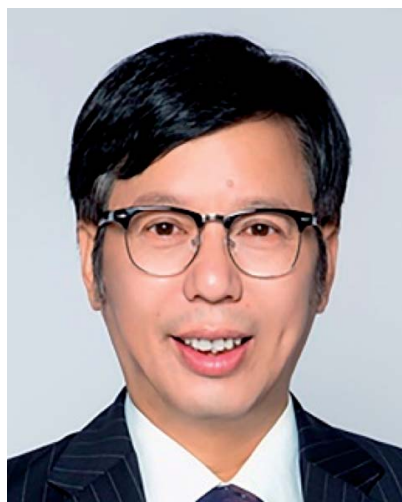

Yi Zhang, PhD, researcher, chief Professor of Chengdu University of TCM, PhD, and M.Sc., tutor, is the director of The Academic Inheritance and Innovation Research Center of Ethnic Medicine of Chengdu University of TCM. At present, he has published more than 200 academic papers. As the leader of the team, he is committed to the modernization of traditional Chinese medicine and ethnic medicine, and his main research direction is the effective material basis and quality control of traditional Chinese medicine and ethnic medicine.

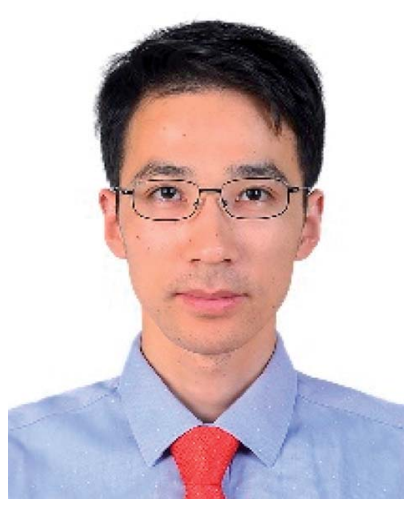

Ce Tang, PhD, post-doctorate, assistant research fellow, currently working at the School of Ethnic Medicine, Chengdu University of TCM. He has undertaken more than 20 projects and published more than 30 research papers, including more than 10 papers in science magazine journals. His research direction is the pharmacodynamic material basis of TCM/ethnic medicine and the development of the key technology of mass spectrometry imaging for the complex traditional Chinese medicine/ethnic medicine system. 
slightly protruding. The flowering and fruit period of $P$. hookeri is from July to September each year. The habitat, the whole plant, and the inflorescence of $P$. hookeri are shown in Fig. 1.

$P$. hookeri mainly grows on hillsides, grasslands, meadows, and forests at an altitude of 1800-5700 m. In China, it is mainly distributed in eastern Tibet, northwestern Yunnan, southern Qinghai, southern Gansu, and northwestern Sichuan. In addition, $P$. hookeri can also be found in Nepal, Sikkim, Bhutan, and northern India. ${ }^{7}$

\section{Traditional medicinal uses}

P. hookeri is mostly used in China and Tibet as a traditional Tibetan medicine, with a long history of its use as a drug. In traditional Tibetan medicine, the main effect of $P$. hookeri is to clear heat, detoxify, dispel wind, and relieve pain. It is mainly used to treat exogenous fever and rheumatoid arthritis. ${ }^{4}$ In the early stages of preparing this paper, the research group referred to Tibetan medicine-related monographs, woodcut boards, and handwritten documents, which confirmed that the earliest record of the application of $P$. hookeri is the Dunhuang Tubo medical document "Chang Juan" (长卷), ${ }^{8}$ which was written before the 7th century AD. Many other ancient Tibetan medical classics, such as “The Four Medical Tantras" (四部医典), "Jing Zhu Materia Medica” (晶珠本草), ${ }^{4}$ and “Lan Liu Li” (蓝琉璃) ${ }^{9}$ also recorded the uses of $P$. hookeri. Moreover, the herb has been included in many standards, such as the Chinese Pharmacopoeia), ${ }^{10}$ Pharmacopoeia standards of the Ministry of Health, ${ }^{11}$ and Tibetan medicine standards ${ }^{\mathbf{1 2}}$ (Fig. 2). In a similar way to traditional Chinese medicine, $P$. hookeri is used to treat diseases in the form of prescriptions. According to the research team's preliminary statistics, 215 prepared preparations containing $P$. hookeri are available, which are mostly used in combination with Corydalis hendersonii, Corydalis mucronifera, Gentiana straminea, and Terminalia chebula for the treatment of plague, pneumonia, colds, measles, biliary fever, intestinal fever, and arthritis. ${ }^{13}$ Some of them have withstood the test of time and are still used by the Chinese Tibetan hospitals, and some have even become mature medicines, occupying a certain place in the market (Table 1). In summary, $P$. hookeri and its prescriptions are mainly used to treat inflammatory and painrelated disease.

\section{Phytochemistry}

To date, 93 phytoconstituents have been isolated from the different parts of $P$. hookeri, including iridoids, triterpenes, fatty acids, lignans, flavonoids, steroids, saccharides, and amino acids (Table 2).

\subsection{Iridoids}

Iridoids are monoterpenoids composed of six-membered oxygen heterocycles and fused cyclo-pentane rings. Iridoids are usually subdivided into four groups: iridoid glycosides, bisiridoids, seco-iridoids, and non-glycosidic iridoids. ${ }^{38-40}$ The main compounds isolated from the different parts of $P$. hookeri are iridoids, and 33 iridoids (Table 2) have been isolated. According to their structures, they were divided into four groups, compounds 1-4 (four iridoids), compounds 5, 6 (two secoiridoids), compounds 7-29 (23 bis-iridoids), and compounds 30-33 (four iridoid oligomers) iridoid glycosides. The structures of these compounds are shown in Fig. 3.

The earliest reports on iridoids in $P$. hookeri were published in 2000. Tian et al. separated loganin (compound 1) from the whole plant of $P$. hookeri for the first time, and the compound was considered the predominant compound. ${ }^{15} \mathrm{Wu}$ et al. reported 24 iridoids (compounds 4, 5, 7-9, 10-28). Compounds 711, 19-20, 27 were originally isolated from $P$. hookeri. The bisiridoids may be the key ingredients which account for the anti-inflammatory effects of $P$. hookeri, and compounds 7, 8, 27 can inhibit TNF- $\alpha$-induced NF- $\kappa$ B-dependent promoter activity. $^{22-25}$ Furthermore, Zhang et al. isolated three iridoid glycosides from the $95 \%$ ethanol extracts from the whole plant of $P$. hookeri in 2014, namely, loganetin (compound 2), 5-[3-(1hydroxyethyl) pyridine], 7-loganin ester (compound 29), and dipsanoside A (compound 30), using chemical methods and spectral analysis. ${ }^{16}$ In addition, Huang et al. isolated two iridoid glycosides (compounds 31, 32) from the 95\% ethanol extracts of

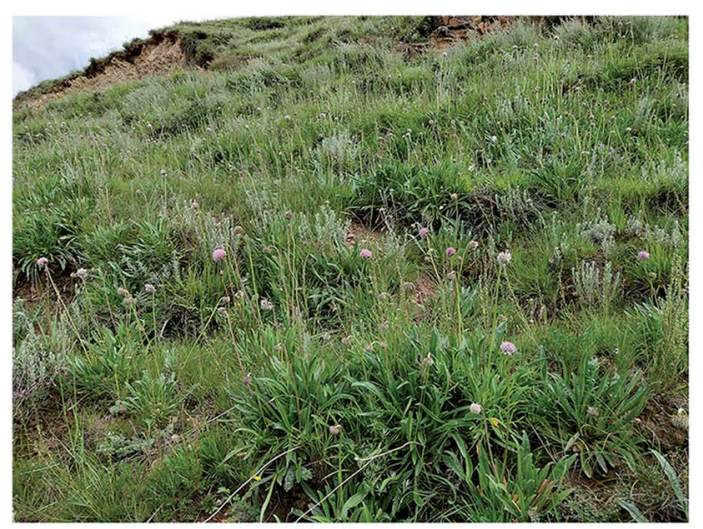

Biotope

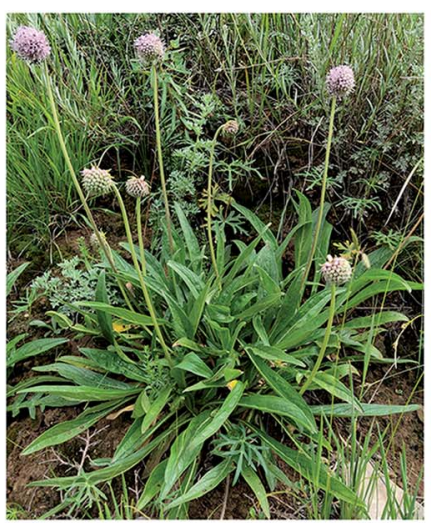

Whole-plant

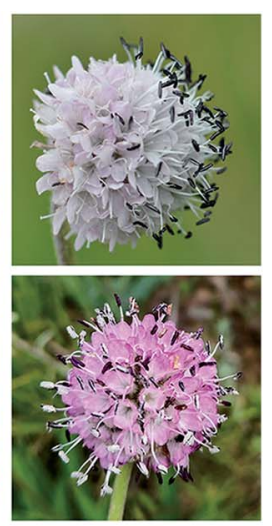

Flower

Fig. 1 Photographs of the original plant of $P$. hookeri in its natural habitat, the whole plant, and its flowers. 


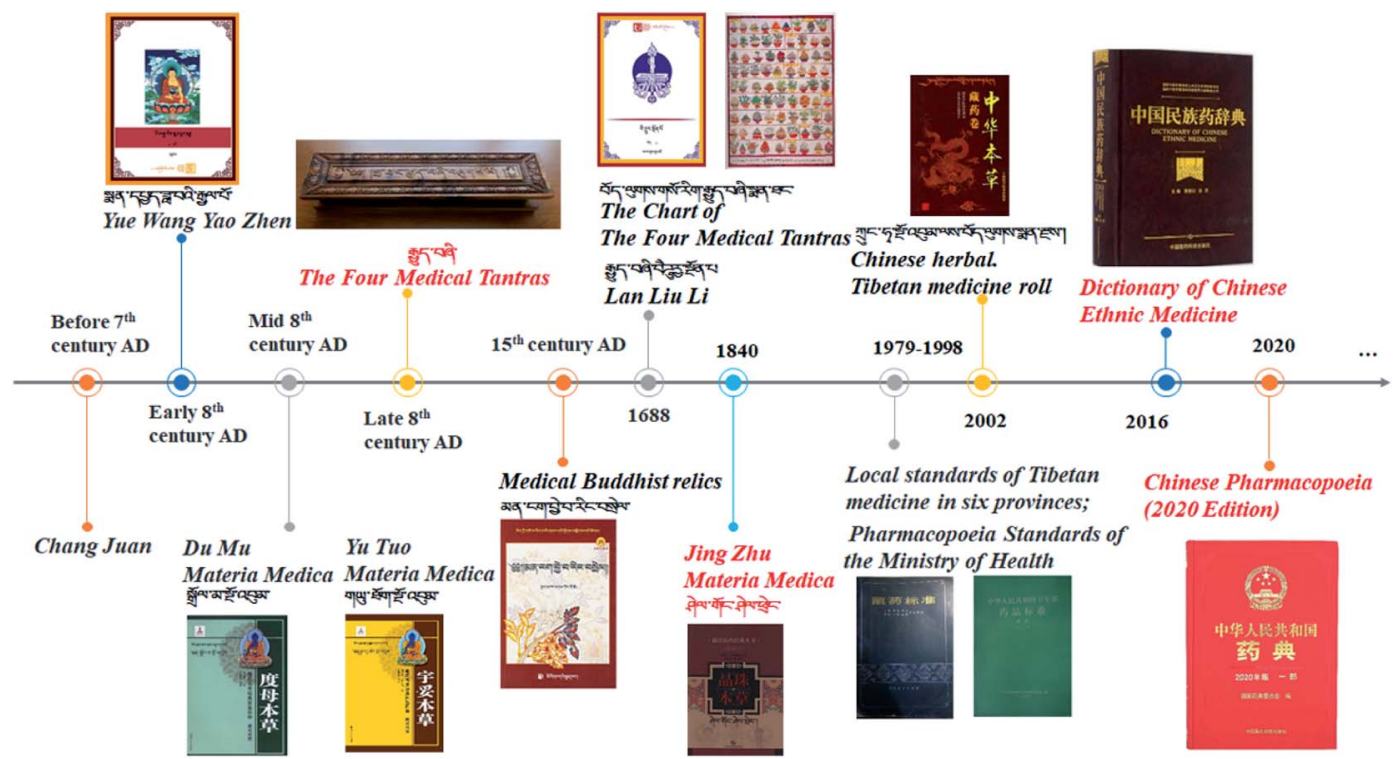

Fig. 2 The important records of $P$. hookeri.

the aerial parts of $P$. hookeri. Compound 32 is a novel iridoid oligomer. Huang et al. elucidated its structure using extensive spectroscopic analysis, including 1D-NMR and 2D-NMR experiments, and showed that it had no significant activity against
MCF-7 (human breast cancer), HEPG2 (human liver carcinoma), and $\mathrm{H} 460$ (human large cell cancer of the lung) cancer cells $\left(\mathrm{IC}_{50}>50 \mu \mathrm{M}, n=3\right) .{ }^{17}$ Moreover, Tang et al. detected two iridoid glycosides (compounds 3, 6) from $70 \%$ methanol extracts

Table 1 Commercial drugs containing $P$. hookeri used in China

\begin{tabular}{|c|c|c|c|c|}
\hline No & Preparation name & Composition & Traditional and clinical uses & Ref. \\
\hline 1 & Shi'erwei Yishou San & $\begin{array}{l}\text { Pterocephali herba, Santali } \\
\text { albi lignum, Carthami flos, } \\
\text { and so on }\end{array}$ & $\begin{array}{l}\text { Treatment of plague, } \\
\text { influenza, Japanese } \\
\text { encephalitis, fever }\end{array}$ & 10 \\
\hline 2 & Jiebai Wan & $\begin{array}{l}\text { Pterocephali herba, Carthami } \\
\text { flos, Myristicae semen, and } \\
\text { so on }\end{array}$ & $\begin{array}{l}\text { Treatment of indigestion, } \\
\text { stomach pain, vomiting and } \\
\text { diarrhea }\end{array}$ & 10 \\
\hline 3 & Pomegranate Puan San & $\begin{array}{l}\text { Pterocephali herba, } \\
\text { Cinnamomi cortex, Piperis } \\
\text { longi fructus, and so on }\end{array}$ & $\begin{array}{l}\text { Treatment of indigestion, } \\
\text { urination problems, } \\
\text { stomach pain }\end{array}$ & 11 \\
\hline 4 & Jiuwei QingPeng San & $\begin{array}{l}\text { Pterocephali herba, Radix } \\
\text { Inulae racemosa, Chebulae } \\
\text { fructus, and so on }\end{array}$ & $\begin{array}{l}\text { Treatment of pneumonia, } \\
\text { fever, sore throat }\end{array}$ & 11 \\
\hline 5 & Dasimabao Wan & $\begin{array}{l}\text { Pterocephali herba, Chebulae } \\
\text { fructus, Aucklandiae radix, } \\
\text { and so on }\end{array}$ & $\begin{array}{l}\text { Treatment of meningitis, } \\
\text { colds, pharyngitis, } \\
\text { pneumonia }\end{array}$ & 11 \\
\hline 6 & Qingfei Zhike Wan & $\begin{array}{l}\text { Pterocephali herba, Arnebiae } \\
\text { radix, Phyllanthi fructus, and } \\
\text { so on }\end{array}$ & $\begin{array}{l}\text { Treatment of lung disease, } \\
\text { colds, cough, chest pain }\end{array}$ & 11 \\
\hline 7 & Ershiwuwei Yuganzi San & $\begin{array}{l}\text { Pterocephali herba, Dendrobii } \\
\text { caulis, Adhatoda vasica Nees, } \\
\text { and so on }\end{array}$ & $\begin{array}{l}\text { Treatment of high blood } \\
\text { pressure, stomach ulcers, } \\
\text { liver pain }\end{array}$ & 11 \\
\hline 8 & Ershiwuwei Yuganzi Wan & $\begin{array}{l}\text { Pterocephali herba, Carthami } \\
\text { flos, Aucklandiae radix, and } \\
\text { so on }\end{array}$ & $\begin{array}{l}\text { Treatment of high blood } \\
\text { pressure, liver pain, thirst, } \\
\text { irregular menstruation }\end{array}$ & 11 \\
\hline 9 & Shierwei Qixiao Tangsan & Pterocephali herba, & Treatment of cough, & 11 \\
\hline
\end{tabular}

Pterocephali herba, Cistanches herba, Tsaoko fructus, and so on other skin diseases 
Table 2 Chemical composition of P. hookeri

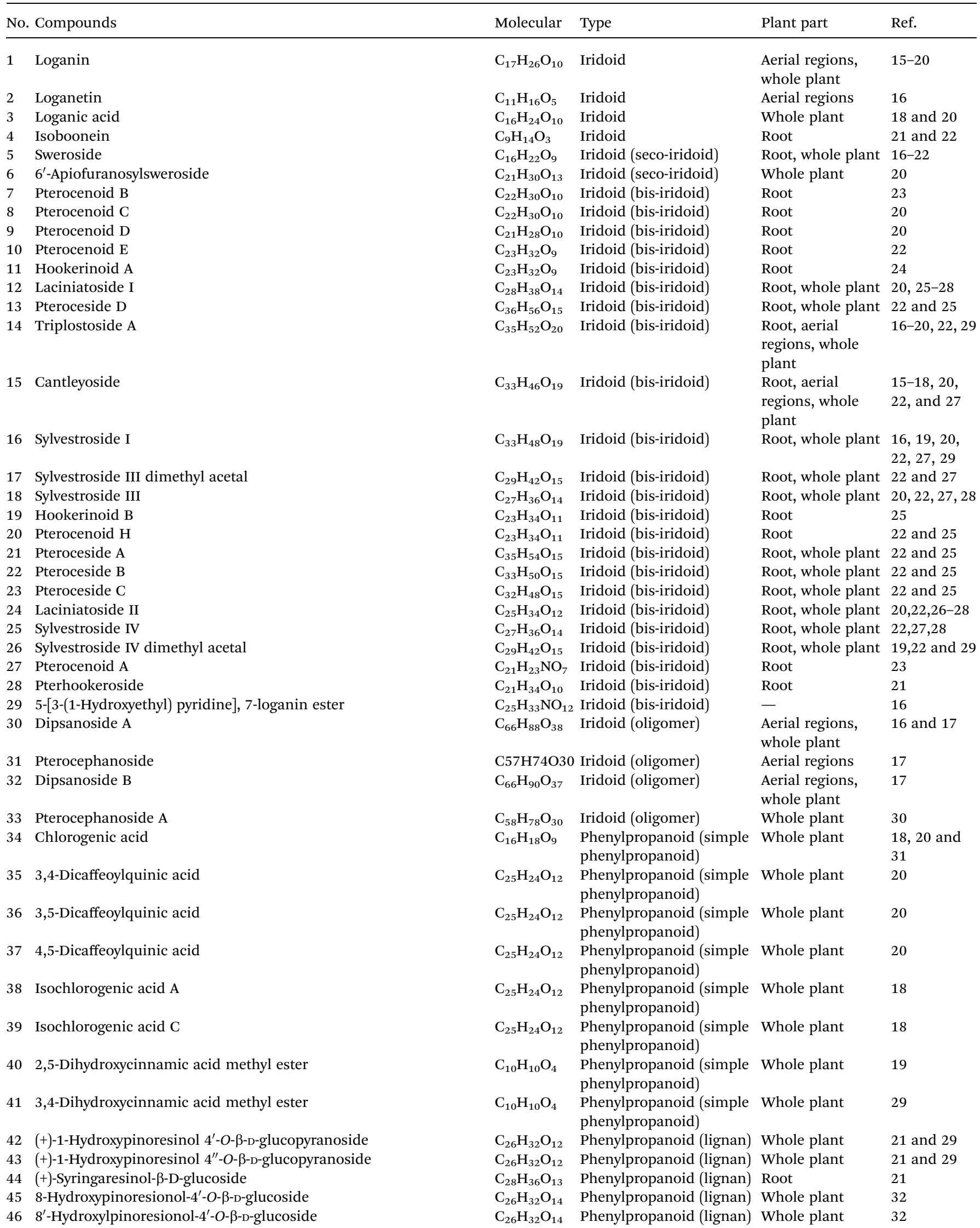


Table 2 (Contd.)

\begin{tabular}{|c|c|c|c|c|c|}
\hline No. & Compounds & Molecular & Type & Plant part & Ref. \\
\hline 47 & $\begin{array}{l}(7 R, 8 S) \text {-Erythro-7,9,9' - trihydroxy-3,3'-dimethoxy-8- } O-4^{\prime} \text {-neolignan-4- } \\
O-\beta \text {-D-glucoside }\end{array}$ & $\mathrm{C}_{26} \mathrm{H}_{36} \mathrm{O}_{12}$ & Phenylpropanoid (lignan) & Root & 21 \\
\hline 48 & Cedrusin-4- $O$ - $\beta$-glucoside & $\mathrm{C}_{26} \mathrm{H}_{34} \mathrm{O}_{11}$ & Phenylpropanoid (lignan) & Root & 21 \\
\hline 49 & Ptehoosine A & $\mathrm{C}_{31} \mathrm{H}_{32} \mathrm{O}_{9}$ & Phenylpropanoid (lignan) & Whole plant & 33 \\
\hline 50 & Ptehoosine B & $\mathrm{C}_{31} \mathrm{H}_{32} \mathrm{O}_{9}$ & Phenylpropanoid (lignan) & Whole plant & 33 \\
\hline 51 & Syringaresinol & $\mathrm{C}_{22} \mathrm{H}_{26} \mathrm{O}_{8}$ & Phenylpropanoid (lignan) & Whole plant & 33 \\
\hline 52 & Pinoresinol & $\mathrm{C}_{20} \mathrm{H}_{22} \mathrm{O}_{6}$ & Phenylpropanoid (lignan) & Whole plant & 33 \\
\hline 53 & Hookeroside A & $\mathrm{C}_{52} \mathrm{H}_{84} \mathrm{O}_{20}$ & $\begin{array}{l}\text { Triterpenoid (oleanane- } \\
\text { type) }\end{array}$ & Whole plant & $\begin{array}{l}6,19,20 \text { and } \\
29\end{array}$ \\
\hline 54 & Hookeroside B & $\mathrm{C}_{57} \mathrm{H}_{92} \mathrm{O}_{24}$ & $\begin{array}{l}\text { Triterpenoid (oleanane- } \\
\text { type) }\end{array}$ & Whole plant & $\begin{array}{l}6,19,20 \text { and } \\
29\end{array}$ \\
\hline 55 & Hookeroside C & $\mathrm{C}_{83} \mathrm{H}_{102} \mathrm{O}_{28}$ & $\begin{array}{l}\text { Triterpenoid (oleanane- } \\
\text { type) }\end{array}$ & Whole plant & $\begin{array}{l}6,19,20 \text { and } \\
29\end{array}$ \\
\hline 56 & Hookeroside D & $\mathrm{C}_{71} \mathrm{H}_{82} \mathrm{O}_{18}$ & $\begin{array}{l}\text { Triterpenoid (oleanane- } \\
\text { type) }\end{array}$ & Root, whole plant & $\begin{array}{l}6,15,19,20 \\
\text { and } 29\end{array}$ \\
\hline 57 & Songoroside A & $\mathrm{C}_{35} \mathrm{H}_{56} \mathrm{O}_{7}$ & $\begin{array}{l}\text { Triterpenoid (oleanane- } \\
\text { type) }\end{array}$ & Whole plant & $\begin{array}{l}6,15,19,20 \\
\text { and } 29\end{array}$ \\
\hline 58 & Oleanolic acid & $\mathrm{C}_{30} \mathrm{H}_{48} \mathrm{O}_{3}$ & $\begin{array}{l}\text { Triterpenoid (oleanane- } \\
\text { type) }\end{array}$ & Whole plant & $\begin{array}{l}6,15,19,20 \\
\text { and } 29\end{array}$ \\
\hline 59 & Rivularicin & $\mathrm{C}_{62} \mathrm{H}_{100} \mathrm{O}_{27}$ & $\begin{array}{l}\text { Triterpenoid (oleanane- } \\
\text { type) }\end{array}$ & Whole plant & $\begin{array}{l}19,20 \text { and } \\
34\end{array}$ \\
\hline 60 & $\begin{array}{l}\text { Oleanolic acid } 3-O-\beta \text {-D-xylopyranosyl }(1 \rightarrow 3)-\alpha-\mathrm{L}^{-} \\
\text {rhammopyranosyl }(1 \rightarrow 2)-\beta \text {-D-xylopyranoside }\end{array}$ & $\mathrm{C}_{43} \mathrm{H}_{56} \mathrm{O}_{19}$ & $\begin{array}{l}\text { Triterpenoid (oleanane- } \\
\text { type) }\end{array}$ & Whole plant & 19 and 29 \\
\hline 61 & $\begin{array}{l}\text { Oleanolic acid } 3-O-\beta \text {-D-xylopyranosyl }(1 \rightarrow 4) \text { - } \beta \text {-D-glucopyranosyl }(1 \rightarrow \\
\text { 2)- } \beta \text {-D-xylopyranoside }\end{array}$ & $\mathrm{C}_{47} \mathrm{H}_{74} \mathrm{O}_{30}$ & $\begin{array}{l}\text { Triterpenoid (oleanane- } \\
\text { type) }\end{array}$ & Whole plant & 19 and 29 \\
\hline 62 & $\begin{array}{l}\text { Oleanolic acid 3-O- } \beta \text {-D-glucopyranosyl }(1 \rightarrow 4)-\beta \text {-D-xylopyranosy }(1 \rightarrow 3) \text { - } \\
\alpha \text {-L-rhamnopyanosyl }(1 \rightarrow 2)-\beta \text {-D-xylopyranoside }\end{array}$ & $\mathrm{C}_{42} \mathrm{H}_{46} \mathrm{O}_{25}$ & $\begin{array}{l}\text { Triterpenoid (oleanane- } \\
\text { type) }\end{array}$ & Whole plant & 19 and 29 \\
\hline 63 & Giganteaside D & $\mathrm{C}_{31} \mathrm{H}_{48} \mathrm{O}_{15}$ & $\begin{array}{l}\text { Triterpenoid (oleanane- } \\
\text { type) }\end{array}$ & Whole plant & $\begin{array}{l}19,20 \text { and } \\
29\end{array}$ \\
\hline 64 & Prosapogenin Ax & $\mathrm{C}_{42} \mathrm{H}_{68} \mathrm{O}_{24}$ & $\begin{array}{l}\text { Triterpenoid (oleanane- } \\
\text { type) }\end{array}$ & Whole plant & 6 and 29 \\
\hline 65 & Prosapogenin Bx & $\mathrm{C}_{47} \mathrm{H}_{75} \mathrm{O}_{30}$ & $\begin{array}{l}\text { Triterpenoid (oleanane- } \\
\text { type) }\end{array}$ & Whole plant & 6 and 29 \\
\hline 66 & Pterocephin A & $\mathrm{C}_{52} \mathrm{H}_{84} \mathrm{O}_{35}$ & $\begin{array}{l}\text { Triterpenoid (oleanane- } \\
\text { type) }\end{array}$ & Whole plant & 35 \\
\hline 67 & Oleanonic acid & $\mathrm{C}_{30} \mathrm{H}_{46} \mathrm{O}_{3}$ & $\begin{array}{l}\text { Triterpenoid (oleanane- } \\
\text { type) }\end{array}$ & Whole plant & 36 \\
\hline 68 & Ursolic acid & $\mathrm{C}_{30} \mathrm{H}_{48} \mathrm{O}_{3}$ & Triterpenoid (ursane-type) & Whole plant & $\begin{array}{l}6,15,19 \text { and } \\
20\end{array}$ \\
\hline 69 & $\begin{array}{l}\text { 11,12-Epoxy-2,6-dihydroxy-24-norursa-1,4-dien-3-on-2-on-(28 } \rightarrow 13) \text { - } \\
\text { olide }\end{array}$ & $\mathrm{C}_{28} \mathrm{H}_{36} \mathrm{O}_{6}$ & Triterpenoid (ursane-type) & Root & 24 \\
\hline 70 & Hookerinoid C & $\mathrm{C}_{28} \mathrm{H}_{40} \mathrm{O}_{5}$ & Triterpenoid (ursane-type) & Root & 24 \\
\hline 71 & Palmitic acid & $\mathrm{C}_{16} \mathrm{H}_{32} \mathrm{O}_{2}$ & Fatty acid (saturated) & Whole plant & 6 and 15 \\
\hline 72 & Heptadecanoic acid & $\mathrm{C}_{17} \mathrm{H}_{34} \mathrm{O}_{2}$ & Fatty acid (saturated) & Whole plant & 37 \\
\hline 73 & Pentadecanoic acid & $\mathrm{C}_{15} \mathrm{H}_{30} \mathrm{O}_{2}$ & Fatty acid (saturated) & Whole plant & 37 \\
\hline 74 & Myristic acid & $\mathrm{C}_{14} \mathrm{H}_{28} \mathrm{O}_{2}$ & Fatty acid (saturated) & Whole plant & 37 \\
\hline 75 & Stearic acid & $\mathrm{C}_{18} \mathrm{H}_{36} \mathrm{O}_{2}$ & Fatty acid (saturated) & Whole plant & 37 \\
\hline 76 & Arachidic acid & $\mathrm{C}_{20} \mathrm{H}_{40} \mathrm{O}_{2}$ & Fatty acid (saturated) & Whole plant & 37 \\
\hline 77 & Behenic acid & $\mathrm{C}_{22} \mathrm{H}_{44} \mathrm{O}_{2}$ & Fatty acid (saturated) & Whole plant & 37 \\
\hline 78 & Lignoceric acid & $\mathrm{C}_{24} \mathrm{H}_{48} \mathrm{O}_{2}$ & Fatty acid (saturated) & Whole plant & 37 \\
\hline 79 & Lignoceric acid & $\mathrm{C}_{23} \mathrm{H}_{46} \mathrm{O}_{2}$ & Fatty acid (saturated) & Whole plant & 37 \\
\hline 80 & Ginkgolic acid & $\mathrm{C}_{22} \mathrm{H}_{34} \mathrm{O}_{3}$ & Fatty acid (unsaturated) & Whole plant & 37 \\
\hline 81 & Linoleic acid & $\mathrm{C}_{18} \mathrm{H}_{32} \mathrm{O}_{2}$ & Fatty acid (unsaturated) & Whole plant & 37 \\
\hline 82 & Oleic acid & $\mathrm{C}_{18} \mathrm{H}_{34} \mathrm{O}_{2}$ & Fatty acid (unsaturated) & Whole plant & 37 \\
\hline 83 & Palmitoleic acid & $\mathrm{C}_{16} \mathrm{H}_{30} \mathrm{O}_{2}$ & Fatty acid (unsaturated) & Whole plant & 37 \\
\hline 84 & Eicosenoic acid & $\mathrm{C}_{20} \mathrm{H}_{38} \mathrm{O}_{2}$ & Fatty acid (unsaturated) & Whole plant & 37 \\
\hline 85 & $\alpha$-Linolenic acid & $\mathrm{C}_{18} \mathrm{H}_{30} \mathrm{O}_{2}$ & Fatty acid (unsaturated) & Whole plant & 37 \\
\hline 86 & $5,7,3^{\prime}, 4^{\prime}, 6^{\prime}$-Pentahydroxyflavanone & $\mathrm{C}_{15} \mathrm{H}_{12} \mathrm{O}_{7}$ & Flavonoid (flavanone) & Whole plant & 36 \\
\hline 87 & Luteolin & $\mathrm{C}_{15} \mathrm{H}_{10} \mathrm{O}_{6}$ & Flavonoid (flavone) & Whole plant & 8 \\
\hline 88 & L-Methionine & $\mathrm{C}_{5} \mathrm{H}_{11} \mathrm{NO}_{2} \mathrm{~S}$ & Amino acid & Whole plant & 36 \\
\hline 89 & p-Hydroxy benzaldehyde & $\mathrm{C}_{7} \mathrm{H}_{6} \mathrm{O}_{2}$ & Phenolic acid & Whole plant & 36 \\
\hline 90 & $\beta$-Gentiobiose & $\mathrm{C}_{12} \mathrm{H}_{22} \mathrm{O}_{11}$ & Carbohydrate & Whole plant & 15 \\
\hline 91 & Twenty alkyl ethers & $\mathrm{C}_{40} \mathrm{H}_{82} \mathrm{O}$ & Ethers & Whole plant & 36 \\
\hline
\end{tabular}


Table 2 (Contd.)

\begin{tabular}{|c|c|c|c|c|}
\hline No. Compounds & Molecular & Type & Plant part & Ref. \\
\hline 92 Pentatriacontane & $\mathrm{C}_{35} \mathrm{H}_{72}$ & Alkane & Whole plant & 36 \\
\hline $93 \beta$-Sitosterol & $\mathrm{C}_{29} \mathrm{H}_{50} \mathrm{O}$ & Steroid & Whole plant & 36 \\
\hline
\end{tabular}

of $P$. hookeri using UPLC-Q-TOF/MS and identified them by using standard products. ${ }^{20}$ Most recently, pterocephanoside A (compound 33) was isolated from $P$. hookeri, and the compound mostly possessed seco-iridoid subtype and iridoid subtype skeletons and showed an inhibitory effect on LPS-induced NO production in RAW 264.7 cells (murine macrophages), but the activity was weak. ${ }^{30}$

\subsection{Phenylpropanoids}

The skeleton of phenylpropanoid is formed from C6-C3 units, however, some of the carbons in the side chain might have been lost during the biosynthesis. Phenylpropanoids are widely found in traditional Chinese medicine and have various physiological activities, such as antioxidation, anti-inflammatory, antibacterial, hemostasis, antitumor, and they can give cardiovascular protection. ${ }^{\mathbf{4 1 - 4 3}}$ To date, 19 phenylpropanoids have been detected this plant, and they are divided into two groups: simple phenylpropanoids (compounds 34-41) and lignans (compounds 42-52), according to their structures, and they are shown in Fig. 4.

Compounds (42-44, 47, 48) were isolated and purified from $P$. hookeri with silica gel chromatography using a Sephadex LH20 column, semipreparative HPLC, NMR, and HR-ESIMS. It should be noted, that these compounds have never been found in the genus Pterocephalus before, and have the potential to become useful chemotaxonomic markers of Pterocephalus. ${ }^{21} \mathrm{Li}$ et al. established a UFLC-PDA fingerprint analysis method for use with $P$. hookeri from different producing areas, and identified five components with standards, including compound $34 .^{31}$ Two phenylpropanoids (compounds 45,46 ) were separated and identified in the $P$. hookeri $95 \%$ ethanol extracts by spectral analyses, including HR-ESIMS, NMR, and HPLC. ${ }^{32}$ Compound 40 and 41 were originally isolated from $P$. hookeri.$^{19,29}$ Tang et al. using a standard comparison method, identified three

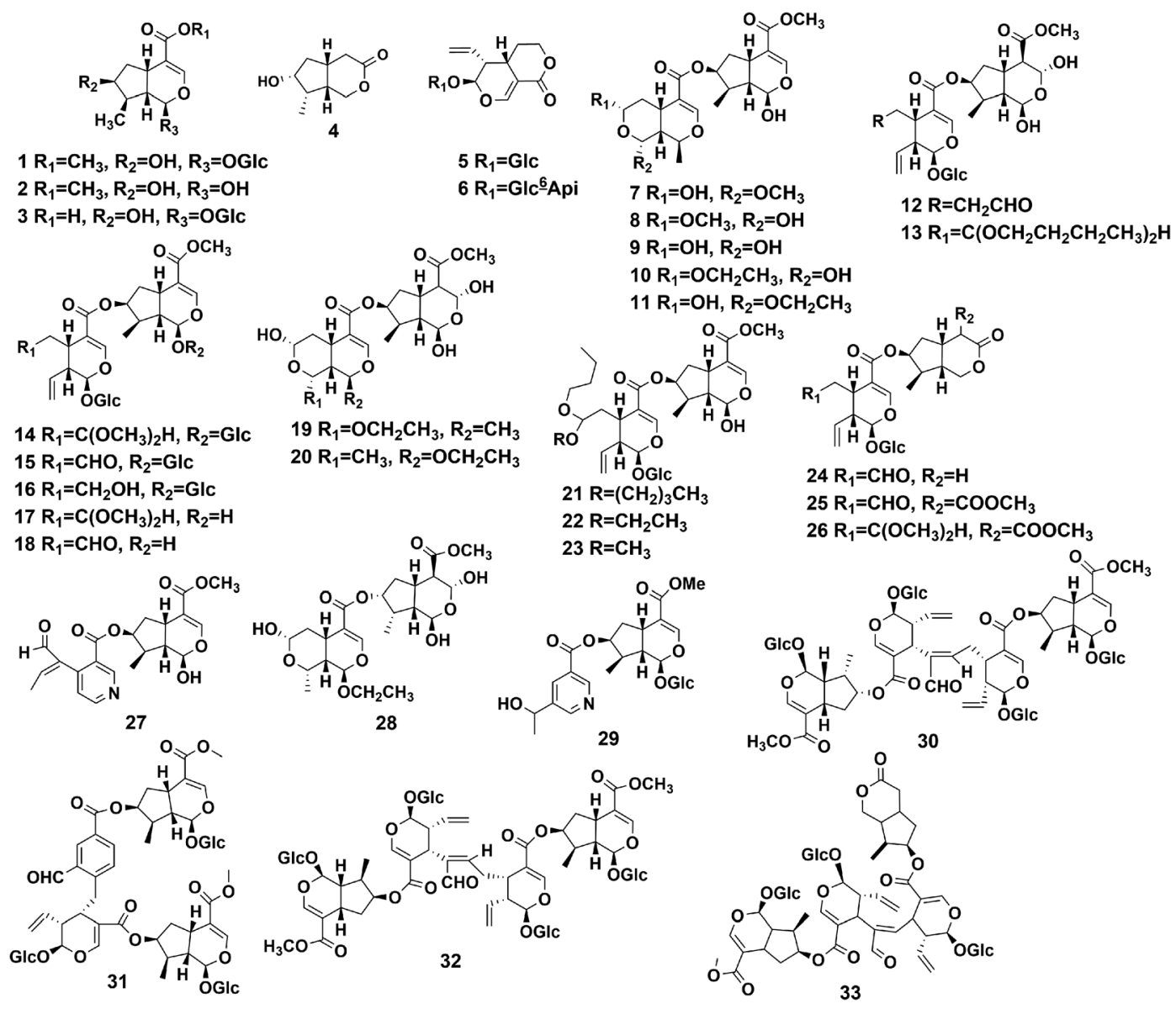

Fig. 3 The structures of the iridoids obtained from P. hookeri. 


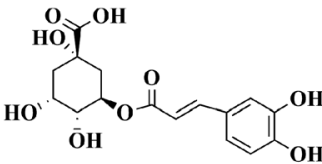

34<smiles>CC(=O)C=Cc1cc(Br)c(Br)c([18OH])c1N</smiles>

$40 R_{1}=R_{4}=\mathrm{OH}, R_{2}=R_{3}=H$ $41 R_{1}=R_{2}=H, R_{2}=R_{3}=O H$

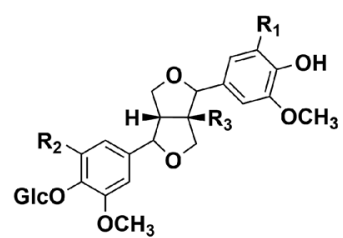

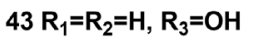
$44 \mathrm{R}_{1}=\mathrm{R}_{2}=\mathrm{OCH}_{3}, \mathrm{R}_{3}=\mathrm{H}$<smiles>C=C[C@H](O)[C@H](O)c1ccc(OC)c(OC)c1OC</smiles>
47<smiles>[R]COc1cc(O)c(OC)cc1-c1cc([C@@H]2CO[C@H](c3cc(OC)c(O)cc3OC)C2)ccc1OC</smiles>

$49 \mathrm{R}=\mathrm{Me}$ $50 \mathrm{R}=\mathrm{H}$<smiles>CCOC(=O)C=Cc1ccc(O)c(O)c1</smiles>

$35 R_{1}=H, R_{2}=$ Caffeoyl, $R_{3}=$ Caffeoyl $36 R_{1}=$ Caffeoyl, $R_{2}=H, R_{3}=$ Caffeoyl $37 \mathbf{R}_{\mathbf{1}}=$ Caffeoyl, $\mathbf{R}_{\mathbf{2}}=$ Caffeoyl, $\mathbf{R}_{\mathbf{3}}=\mathrm{H}$ $38 R_{1}=$ Caffeoyl, $R_{2}=H, R_{3}=$ Caffeoyl $39 \mathrm{R}_{1}=$ Caffeoyl, $\mathrm{R}_{2}=$ Caffeoyl, $\mathrm{R}_{3}=\mathrm{H}$

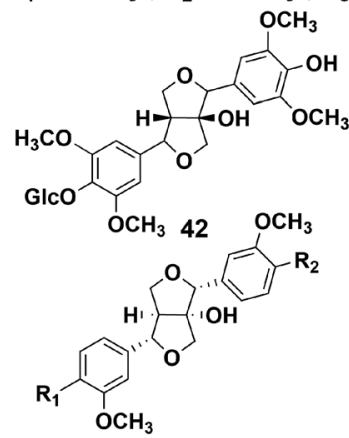
$45 \mathrm{R}_{1}=$ B-D-Glc, $\mathrm{R}_{2}=\mathrm{OH}$ $46 \mathrm{R}_{1}=\mathrm{OH}, \mathrm{R}_{2}=ß-\mathrm{D}-\mathrm{GlC}$<smiles>COc1cc(C2Oc3cc(c(OC)cc3CCCO)C2CO)ccc1Cl</smiles>

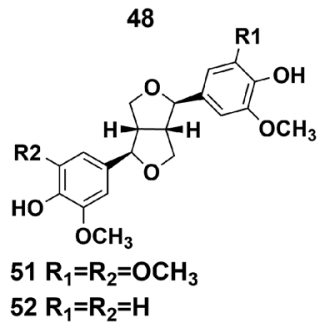

Fig. 4 The skeletal structures of phenylpropanoids obtained from $P$. hookeri.

phenylpropyl compounds (compounds 35-37) from a 70\% methanol extract of $P$. hookeri using UPLC-Q-TOF/MS. ${ }^{20}$ Meanwhile, Wang et al. used UPLC-PDA to identify compounds 38, 39 from a methanol extract of $P$. hookeri, and proved that these compounds could be absorbed by rats using the everted intestinal sac model. ${ }^{18}$ Two undescribed lignans (compounds 49, 50) and two known lignans (compounds 51, 52) were isolated by Dong et al. ${ }^{33}$ Compound $\mathbf{4 9}$ was found to be effective in inhibiting angiogenesis. ${ }^{32}$

\subsection{Triterpenoids}

Terpenoids represent the largest and most diverse class of natural products produced by plants. ${ }^{44}$ Terpenoids are abundant in $P$. hookeri and play an important role in many important physiological activities and biological functions. The main terpenoids in $P$. hookeri are two types of triterpenoids, including the oleanane type (compounds 53-67) and the ursane type (compounds 68-70). A total of 18 triterpenoids have been

reported, and most of them share the same skeleton as the oleanane type. One of the characteristics of the oleanane type pentacyclic triterpenoids of $P$. hookeri is their aglycones, which are always replaced by glycosyl groups at the 3 or 28 sites. Their structures are shown in Fig. 5.

Using DA 201 resin, silica gel, and spectroscopic analysis methods, such as IR and NMR, Tian et al. separated and identified seven triterpenoids (compounds 53-58, 68) from the $95 \%$ ethanol extracts of the whole plant of $P$. hookeri. This study was the first to isolate these compounds from P. hookeri. ${ }^{6}$ In 2002, Zhang et al. isolated rivularicin (compound 59) from P. hookeri for the first time. ${ }^{34}$ They also separated oleanonic acid (compound 67) from the 95\% ethanol extracts of the whole plant of $P$. hookeri, and then established a quality control method for $P$. hookeri analysis using the oleanolic acid content. In addition, they verified that oleanonic acid exerts inhibitory effects on Staphylococcus aureus, Escherichia coli, and Bacillus subtilis. ${ }^{36}$ Another two triterpenoids (compounds 69, 70) were isolated and identified, and compound 69 was found to significantly inhibit the proliferation of Hep3B cells (human hepatocyte carcinoma), with an $\mathrm{IC}_{50}$ of $17.06 \mu \mathrm{m} .{ }^{22} \mathrm{Yu}$ et al. isolated and purified four triterpenoids (compounds 60-63) with silica gel and Sephadex LH-20 columns and then determined their spectral data. ${ }^{19}$ Zhang et al. studied the chemical components in the $90 \%$ ethanol extracts of $P$. hookeri using physical and chemical properties obtained by spectral data analysis and identified another two triterpenoids (compounds 64, 65). ${ }^{29}$ Wang et al. isolated pterocephin A (compound 66) from $P$. hookeri. According to published pharmacological studies, the survival rates of L-02 cells (human fetal hepatocytes) decreased significantly when treated with pterocephin A at a concentration of $16 \mu \mathrm{mol} \mathrm{L} \mathrm{L}^{-1}$, showing that the compound has obvious cytotoxicity. ${ }^{35}$

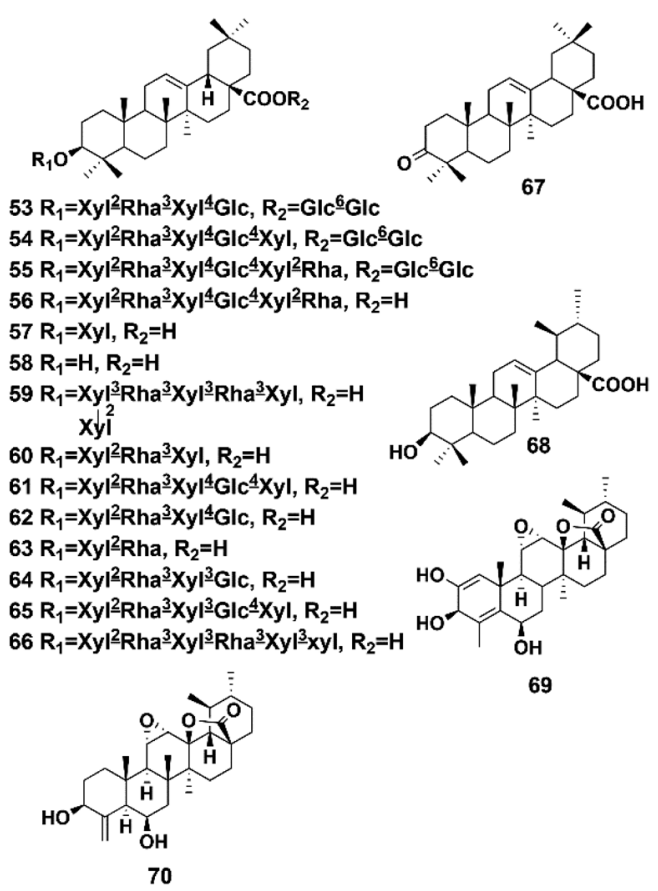

Fig. 5 The structures of triterpenoids obtained from $P$. hookeri. 


\subsection{Fatty acids}

Fatty acids, used to be considered as only energy sources and structural components of the cell membrane, but they now show great potential for the treatment of several diseases, such as type II diabetes mellitus, nonalcoholic steatohepatitis, and chemically induced liver injury. ${ }^{\mathbf{4 5 - 4 7}}$ A total of $\mathbf{1 5}$ fatty acids have been isolated from $P$. hookeri, and these were divided into two group: saturated fatty acids (compounds 71-79) and unsaturated fatty acid (compounds 79-85), and their structures are shown in Fig. 6.

Palmitic acid (compound 71) was the first reported fatty acid found in P. hookeri. ${ }^{15}$ Then, by using GC-MS, Zhang et al. detected 14 fatty acid components (compounds 72-85) from $P$. hookeri at the flowering and nonflowering stages. The compounds were identified by comparison with standards. Moreover, $\alpha$-linolenic acid, a plant-derived n-3 polyunsaturated fatty acid, is a potential fatty acid biomarker because it is useful in distinguishing between the two groups. ${ }^{36,37}$

\subsection{Other compounds}

Some compounds do not belong to the previous classifications, such as flavonoids, carbohydrates, and steroids. Their structures are shown in Fig. 7.

In 2013, Zhang et al. isolated six compounds (compounds 86, $\mathbf{8 8}, \mathbf{8 9}, \mathbf{9 1 - 9 3}$ ) from a $95 \%$ ethanol extract of a whole $P$. hookeri plant and identified them with chemical methods and spectral analysis. Compound $\mathbf{8 6}$ was found to have a significant inhibitory effect on Staphylococcus aureus. ${ }^{36}$ The only carbohydrate isolated from $P$. hookeri is $\beta$-gentiobiose (compound 90). ${ }^{15}$ In addition to compound $\mathbf{8 6}$, another flavonoid, luteolin (compound 87), was separated from the whole plant of $P$. hookeri. ${ }^{29}$ It is a common ingredient with a wide range of

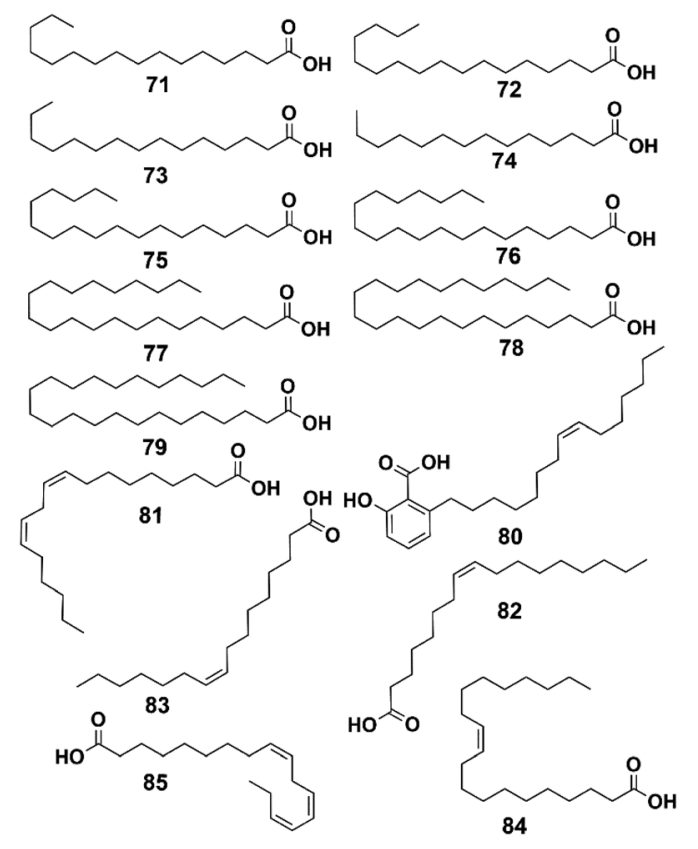

Fig. 6 The structures of fatty acids obtained from $P$. hookeri.

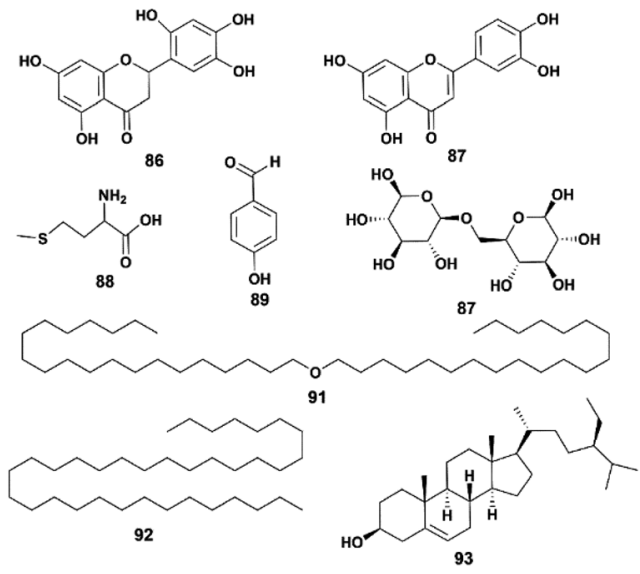

Fig. 7 The structures of compounds 86-93 obtained from $P$. hookeri.

pharmacological activities, such as antitumor, lipid lowering, anti-inflammatory and antioxidant activities. ${ }^{4-50}$

\section{Pharmacological activities}

P. hookeri is in widespread use, and its extracts and chemical components have been used in in vitro and in vivo experimental models. All its pharmacological activities are summarized in Fig. 8 and the following subsections will describe these in more detail.

\subsection{Anti-inflammatory activity}

The primary anti-inflammatory evaluation of $P$. hookeri was based mainly on the mouse models of xylene-induced ear edema and acetic acid-induced peritoneal capillary permeability, and rat models of carrageenan/fresh egg white-induced paw edema and cotton/agar pellet granuloma. In 2004, Guan et al. evaluated the anti-inflammatory activity of an $n$-butanol extract of $P$. hookeri, and the results showed that the extract had significant inhibitory effects on xylene-induced ear edema in mice and fresh egg white-induced paw edema in rats $(P<0.05)$ when the dose was $0.25-0.7 \mathrm{~g} \mathrm{~kg}^{-1}{ }^{51}$ Interestingly, Zhang et al. (2009), Shen et al. (2017), and Chen et al. (2018) reported similar results. $^{27,52,53}$ Another model of acute inflammation is acetic acid-induced peritoneal capillary permeability. Acetic acid can increase the levels of prostaglandins, histamines, 5-hydroxytryptamine and other chemical mediators in the abdominal fluid, thus increasing vascular permeability. Using an ethanol extract of $P$. hookeri $\left(2 \mathrm{~g} \mathrm{~kg}^{-1}\right)$, water extract $\left(4 \mathrm{~g} \mathrm{~kg}^{-1}\right)$, and total glycosides $\left(28-112 \mathrm{mg} \mathrm{kg}^{-1}\right.$, the main components found are compounds $1,5,15,16)$ which can significantly inhibit the increase in vascular permeability induced by acetic acid in mice. The cotton/agar pellet granuloma model and dry weight of chronic inflammation correlated with the level of granulomatous tissue formation. An ethanol extract of $P$. hookeri $\left(2 \mathrm{~g} \mathrm{~kg}^{-1}\right)$, $n$-butanol extract $\left(0.25-0.5 \mathrm{~g} \mathrm{~kg}^{-1}\right)$, and total glycosides (28$112 \mathrm{mg} \mathrm{kg}^{-1}$ ) could effectively inhibit cotton ball granuloma. Therefore, in addition to the acute stage of inflammation, the extracts of $P$. hookeri showed anti-inflammatory activity in the chronic stage of inflammation as well. ${ }^{52,53}$ Bis-iridoids from $P$. 


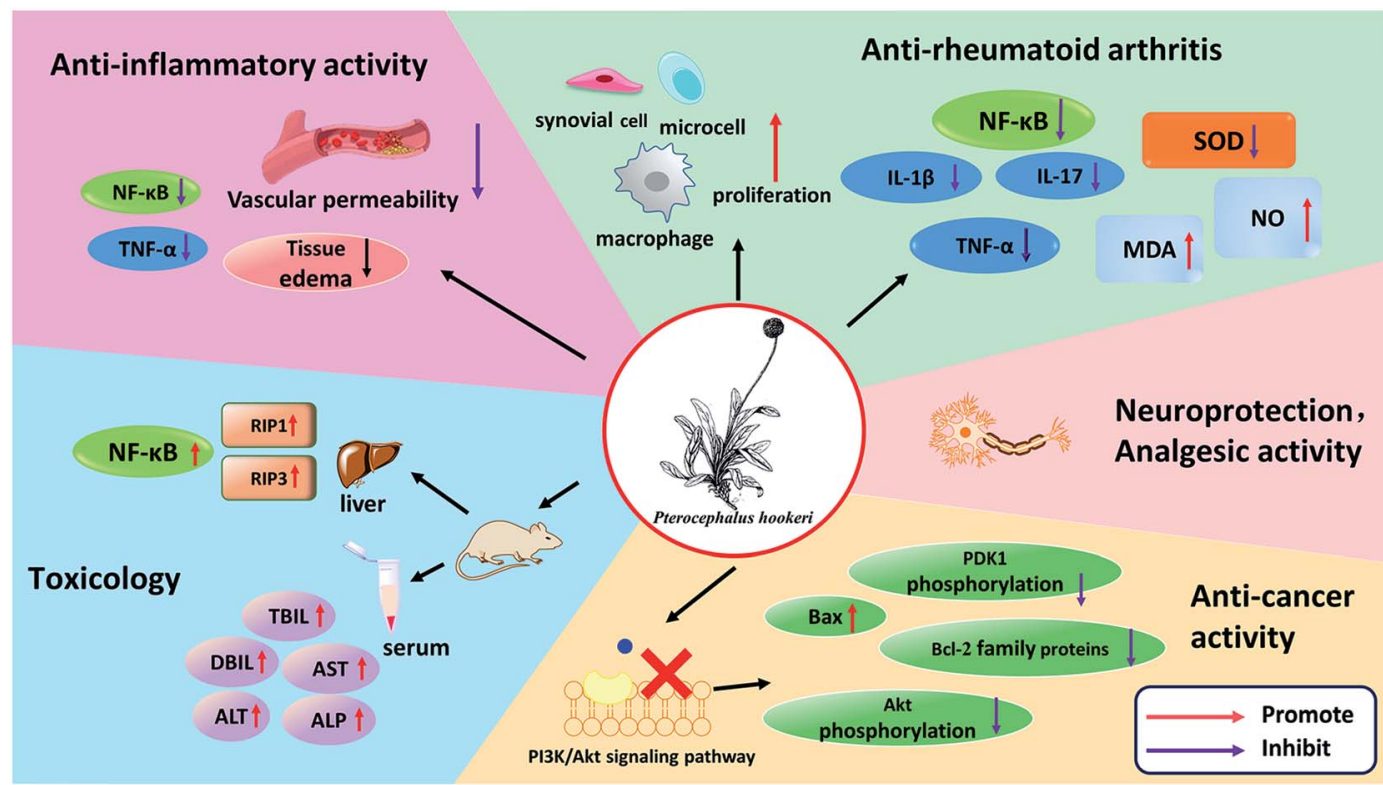

Fig. 8 Overview of the pharmacological activities of $P$. hookeri.

hookeri (25 and $50 \mu \mathrm{M}$, the main components were compounds 11 and 19) significantly reduced TNF- $\alpha$ - and LPS-induced NF- $\kappa$ B activation in HEK293 cells (human embryonic kidney), and the main components, compounds 12, 15-18, 24-26, reduced the production of inflammatory cytokines in a dose-dependent manner. ${ }^{24,27}$

\subsection{Anti-rheumatoid arthritis}

Rheumatoid arthritis (RA) is chronic auto-immune disorder, its main characteristics are swelling and pain in joints, and in synovial membranes. The main treatment is to suppress inflammation and relieve pain. ${ }^{54}$ In Tibetan medicine, RA is known as "zhen bu" disease, and the clinical efficacy of Tibetan medicine on RA is as high as $94.6 \% .^{55} P$. hookeri is commonly used as a treatment for RA in Tibet. The animal adjuvant arthritis (AA) model is a contraindicated animal model mediated by T-cell immunity. ${ }^{56-59}$ It is a commonly used animal model of RA in clinics because of its simplicity in modeling and consistency with clinical manifestations, pathomorphology, and immunological changes in patients with RA. ${ }^{60}$ This model has been adopted in studies on the anti-arthritis effects of $P$. hookeri. Shen et $a .^{53}$ and Yang et al. ${ }^{56}$ investigated the efficacy and mechanism of using the total glucosides of $P$. hookeri on AA rats and found that the total glycosides of $P$. hookeri $(28,56,116$, and $232 \mathrm{mg} \mathrm{kg}^{-1}$ ) significantly reduced primary paw swelling, secondary paw swelling, and arthritis scores in the later stages of AA. In addition, the total glucosides of $P$. hookeri not only improved the proliferation of synovial cells, macrophages, and microcells, and induced inflammatory cell infiltration but it also significantly reduced the levels of IL-1 $\beta$, TNF- $\alpha$, and IL-17. However, these effects were not dose-dependent. Notably, the total glycosides of $P$. hookeri not only significantly increased MDA and NO levels but also significantly decreased the SOD level and the expression of NF- $\kappa \mathrm{B}$ p65 in the synovial tissues of the joints. This result suggested that the anti-RA effect of total glycosides of $P$. hookeri may be related to the antioxidant effect and the inhibition of the NF- $\kappa \mathrm{B}$ signaling pathway. ${ }^{53,56}$

\subsection{Analgesic activity}

The analgesic effects of $P$. hookeri were studied using several types of pain models, including acetic acid-induced abdominal writhing reflex (peripheral pain) and pain caused by a hot plate in mice (central pain). In 2017, the analgesic abilities of total glycosides from $P$. hookeri were evaluated by Shen et al. ${ }^{53}$ At doses of 56 and $112 \mathrm{mg} \mathrm{kg}^{-1}$, obvious analgesic effects on pain induced by acetic acid were observed. However, in a hot-plate test, the pain threshold only increased $30 \mathrm{~min}$ after treatment with $112 \mathrm{mg} \mathrm{kg}^{-1}$ dose. The total glycosides from $P$. hookeri showed a good peripheral analgesic effect, but the central analgesic effect was not obvious. The peripheral analgesic effect may be related to $P$. hookeri's good anti-inflammatory effect. ${ }^{53}$ Moreover, Chen et al. ${ }^{27}$ showed that the bis-iridoid constituents from $P$. hookeri (50 and $100 \mathrm{mg} \mathrm{kg}^{-1}$ ) significantly increased the hot-plate pain threshold and reduced the acetic acid-induced writhing response in mice $(P<0.01)$, and the efficacy of highdose bis-iridoid constituents from $P$. hookeri $\left(100 \mathrm{mg} \mathrm{kg}^{-1}\right.$ ) was superior to that of the positive control (rotundine, $20 \mathrm{mg}$ $\mathrm{kg}^{-1}$ ). The results are similar to those of Zhang's ${ }^{52}$ previous experiment. These results indicated that iridoids possess analgesic effects. ${ }^{27,52}$

\subsection{Anti-cancer activity}

Cancer is not only a serious threat to people's health but also a difficult and hot topic of research. $P$. hookeri has antitumor effects. The total saponins of $P$. hookeri have inhibitory effects on a variety of cancer cells, such as SGC-7901 (human gastric cancer cells), HepG2, AGS gastric adenocarcinoma cells from a human stomach, and MBA-MD-231 (human breast cancer cells).$^{61}$ In 2015, Guo et al. conducted an in-depth study on the 
antitumor effect of an $n$-butanol extract of $P$. hookeri (the main components were compounds 15, 16, 18, 24, 25) on Нер3В cancer cells and found that the extract can selectively inhibit the proliferation of Hep3B cells in vitro, induce apoptosis, block the PI3K pathway, and regulate the protein level of the Bcl-2 family. In addition, the extract inhibited tumor growth by regulating the expression of Bcl-2 family proteins in xenografted tumor mouse models. ${ }^{28,62}$

\subsection{Neuroprotection}

Parkinson's disease, also known as 'wobbly paralysis' in China, is a chronic neurodegenerative disease caused by extrapyramidal dysfunction. The disease usually occurs in middle age or later. The main cause of Parkinson's disease is the damage of dopaminergic neurons in the substantia nigra of the brain, and a decrease in dopamine biosynthesis in the striatum, which results in a significant reduction in dopamine transmitters and hypercholinergic nerve function, and these result in movement disorders. The neuroprotective effects of a $n$-butanol extract of P. hookeri (the main components are compounds 13-18, 24, 25) have been demonstrated in transgenic zebrafish models. The DAT-GFP fertilized eggs treated with $100 \mu \mathrm{g} \mathrm{mL}{ }^{-1} n$-butanol extract were able to resist damage from to $\mathrm{H}_{2} \mathrm{O}_{2}$, and the number of dopamine neurons in the fertilized eggs was the same as that in the control group. ${ }^{26}$

\section{Toxicology}

Although $P$. hookeri has been used as a traditional Tibetan medicine for a long time, its potential toxicity has not been systematically elucidated. According to the records from the classical ancient books, $P$. hookeri has always been considered as a drug with a low toxicity, but reports about its toxicity are few. In early toxicity tests, mice received an oral water extract of $P$. hookeri 3000 times the clinical dose. The mice ate less and showed considerable weight loss. These results indicated that $P$. hookeri had some level of toxicity, but the study was not thorough. ${ }^{51}$

In view of the previous research results, Wang et al. evaluated the hepatotoxicity of an $n$-butanol extract of $P$. hookeri in 2019 and found that the serum levels of ALP, ALT, AST, DBIL, and TBIL were significantly increased $(P<0.05, P<0.01)$ in the mice in the group which had received an $n$-butanol extract of $P$. hookeri. The results showed that the extract caused a certain degree of liver damage in the mice. The expression levels of NF$\kappa \mathrm{B}, \mathrm{RIP} 1$, and RIP3 in the liver tissues and L-02 cells were upregulated after treatment with the extract and the L-02 hepatocytes. These results indicated that the extract can induce liver toxicity in vivo and in vitro, which can induce the development of inflammation and subsequent necrosis. ${ }^{63}$

\section{Qualitative and quantitative analysis}

The most recent quality control standard for $P$. hookeri is in the 2020 edition of the Chinese Pharmacopoeia, in which microscopic and TLC identification is mainly used for qualitative analysis, and quantitative analysis mainly uses HPLC. ${ }^{\mathbf{1 0}}$ Before 2013, a large number of studies showed that $P$. hookeri mainly contains triterpenoid saponins, and oleanolic acid and ursolic acid were considered to be the main quality control components of $P$. hookeri. Therefore, a variety of methods for the determination of oleanolic acid and ursolic acid were established, such as HPLC-evaporative light-scattering detection, HPLC with a photodiode array, and capillary zone electophoresis. ${ }^{37,64-66}$ In 2018, a UPLC-Q-TOF/MS method was established for the analysis of the chemical constituents of $P$. hookeri. A total of 17 iridoid glycosides, 7 phenolic acids, 13 triterpenes, and 3 other components were identified or preliminarily deduced. The 10 main components found were dipsanoside B, sweroside, cantleyoside, chlorogenic acid, loganic acid, loganin, sylvestroside I, dipsanoside A, isochlorogenic acid $\mathrm{A}$, and isochlorogenic acid $\mathrm{C}$, which were quantified using UPLC-PDA. Notably, iridoid glycosides and phenolic acids were found to be the main active components of the 40 compounds identified using molecular docking. The current quality control standard of $P$. hookeri uses oleanolic acid and ursolic acid as quality control indices, which cannot effectively control the quality of the medicinal material, lack direct correlation with biological activity, and are insufficient in evaluating the quality of $P$. hookeri. Therefore, a more suitable and feasible method is needed to comprehensively evaluate the quality of $P$. hookeri. ${ }^{20,67}$

\section{Conclusion and perspectives}

P. hookeri is not only a typical plateau herbaceous plant but also one of the commonly used Tibetan medicines for clearing heat, stopping dysentery, detoxification, eliminating distemper, dispelling wind, and relieving stagnation. This paper reviews the progress of research on $P$. hookeri from the aspects of traditional use, phytochemistry, pharmacology, and toxicology. Because of limited resources and investment, the study of $P$. hookeri and its decoctions have not received enough attention, and the study of its pharmacological activities and related molecular mechanisms is insufficient.

The chemical structures of the compounds found in $P$. hookeri plants are structurally diverse, and mainly include triterpenes, iridoid glycosides, phenolic acids, flavonoids, and other compounds. The crude extract and chemical components of monomers isolated from the plants are still in the preliminary stage, but they do show certain anti-inflammatory, antitumor, neuroprotective, and anti-rheumatoid arthritis activities. However, the identity of the pharmacodynamic substance is still unclear. Modern phytochemical and pharmacological studies have provided some evidence of some of the mechanisms of the action of $P$. hookeri and demonstrate its further development potential as an anti-inflammatory and antitumor agent.

In addition, quality markers from $P$. hookeri are necessary for the study of the mechanisms of action and quality control of medicinal materials. Currently, only oleanolic acid and ursolic acid have been selected as the quality indicators of $P$. hookeri in 
the Chinese Pharmacopoeia in 2020. However, these compounds cannot effectively control the quality of $P$. hookeri. Therefore, chemicals and biomarkers that can better reflect the quality of $P$. hookeri are needed.

In conclusion, long-term clinical practice has proven the safety and efficacy of $P$. hookeri. To fully explore the medicinal value of $P$. hookeri, modern advanced research techniques (mass spectroscopy imaging technology and patch clamp technology) should be used to systematically study its absorption, distribution, metabolism, excretion, tissue distribution, mechanism of action, and quality evaluation, for the development of the use of $P$. hookeri.

\section{Abbreviations}

$\begin{array}{ll}\text { TNF- } \alpha & \text { Tumor necrosis factor- } \alpha \\ \text { NF- } \kappa & \text { Nuclear factor kappa-B } \\ \text { NMR } & \text { Nuclear magnetic resonance } \\ \text { UPLC-Q- } & \text { Ultra-performance liquid chromatography } \\ \text { TOF/MS } & \text { coupled with time-of-flight mass spectrometry } \\ \text { LPS } & \text { Lipopolysaccharide } \\ \text { NO } & \text { Nitric oxide } \\ \text { HPLC } & \text { High-performance liquid chromatography } \\ \text { HR-ESIMS } & \text { High resolution-electrospray ionization mass } \\ & \text { spectrometry } \\ \text { UFLC-PDA } & \text { Ultra-flow liquid chromatography-photo diode } \\ & \text { array } \\ \text { IR } & \text { Infrared spectroscopy } \\ \text { IC } & \text { 50\% inhibition concentration } \\ \text { GC-MS } & \text { Gas chromatography-mass spectrometry } \\ \text { RA } & \text { Rheumatoid arthritis } \\ \text { AA } & \text { Adjuvant arthritis } \\ \text { IL-1 } \beta & \text { Interleukin-1 } \beta \\ \text { IL-17 } & \text { Interleukin-17 } \\ \text { MDA } & \text { Malonaldehyde } \\ \text { SOD } & \text { Superoxide dismutase } \\ \text { Bcl-2 } & \text { B-cell lymphocytoma-2 } \\ \text { ALT } & \text { Alanine aminotransferase } \\ \text { AST } & \text { Aspartate aminotransferase } \\ \text { ALP } & \text { Alkaline phosphatase } \\ \text { DBIL } & \text { Direct bilirubin } \\ \text { TBIL } & \text { Total bilirubin } \\ \text { TLC } & \text { Thin layer chromatography } \\ \text { Glc } & \text { Glucose } \\ \text { Xyl } & \text { Xylose } \\ \text { Rha } & \text { Rhamnose } \\ \text { Api } & \text { Apidside } \\ & \end{array}$

\section{Author contributions}

Zhiqiang Gan - resources, supervision, visualization, writing original draft, writing - review and editing. Juan Jiang - writing - original draft, writing - review and editing. Honglin Tao resources, writing - original draft. Shiying Luo - resources. Xianli Meng - supervision. Jia Yu - supervision, writing - review and editing, funding acquisition. Yi Zhang - supervision, funding acquisition. Ce Tang - resources, supervision, writing original draft, writing - review and editing, funding acquisition.

\section{Conflicts of interest}

The authors declare no conflict of interest.

\section{Acknowledgements}

The authors gratefully acknowledge the financial support from the National Natural Science Foundation of China (No. 81903922 and 81803851), the National Key Research and Development Program of China (No. 2017YFC1703900), and the "Xinglin Scholars" Research Promotion Program of Chengdu University of Traditional Chinese Medicine (BSH2019002).

\section{References}

1 R. Verlaque, A biosystematic and phylogenetic study of the dipsacaceae, Plant Biosystematics, 1984, pp. 307-320.

2 S. E. Carlson, V. Mayer and M. J. Donoghue, Phylogenetic relationships, taxonomy, and morphological evolution in dipsacaceae (dipsacales) inferred by dna sequence data, Taxon, 2009, 58, 1075-1091.

3 Y. N. Li, The Four Medical Tantras, People's Medical Publishing House, Beijing, China, 1983, p. 409.

4 S. D. Luo, Xin Xiu Jing Zhu Materia Medica. Sichuan Science and Technology Press, Sichuan, 2002, p. 591.

5 Committee for the Pharmacopoeia of PR China, Pharmacopoeia of the People's Republic of China, China Medical Science and Technology Press, Beijing, China, 1977.

6 J. Tian, F. E. Wu, M. H. Qiu and R. L. Nie, Triterpenoid Sanponins from Pterocephalus hookeri, Phytochemistry, 1993, 32, 1535-1538.

7 Flora of China Editorial Committee, Flora of China, 2011, vol. 19, pp. 655-656.

8 B. F. Luo, Tubo medical literature of Dunhuang "long scroll", China Academic Journal Electronic Publishing House, 2002, vol. 3, pp.74-86.

9 S. J. J. C. Disi, Lan Liu Li, Shanghai Scientific \& Technical Publishers, Shanghai, China, 2012, p. 137.

10 Committee for the Pharmacopoeia of PR China, Pharmacopoeia of the People's Republic of China (I), China Medical Science and Technology Press, Beijing, China, 2020.

11 Chinese Pharmacopoeia Commission of the Ministry of Public Health, Pharmacopoeia Standards of the Ministry of Health, P.R. China (Tibetan Medicine), Ministry of Public Health, P.R. China, Beijing, 1995, pp. 139-317.

12 Health bureaus of Tibet, Qinghai, Sichuan, Gansu, Yunnan and Xinjiang provinces and Local standards of Tibetan medicine in six provinces, Tibetan medicine standards, Qinghai People's Publishing House, Qinghai, China, 1979, p. 110.

13 F. Zuo, Z. C. Wei, C. Tang, W. Q. Wang, D. Tong, X. L. Meng and Y. Zhang, Medication rules for prescriptions containing Pterocephali Herba based on data mining, China J. Chin. Mater. Med., 2017, 42, 3213-3218. 
14 National Medical Products Administration, National Chinese patent medicine standard compilation ophthalmology otolaryngology dermatology volume, National Medical Products Administration, 2002, p. 346.

15 J. Tian, F. E. Wu, M. H. Qiu and R. L. Nie, Chemical constituents of Pterocephalus hookeri, Nat. Prod. R\&D, 2000, 01, 35-38.

16 J. F. Zhang, S. Huang, L. Z. Shan, L. Chen, Y. Zhang and X. L. Zhou, Studies on the chemical constituents of Tibetan medicine Pterocephalus hookeri, Proceedings of the 10th National Natural Organic Chemistry Conference of the Chinese Chemical Society, 2014, vol. 1, p. 241.

17 S. Huang, J. F. Zhang, L. H. Shan, Y. Zhang and X. L. Zhou, A Novel Tetrairidoid Glucoside from Pterocephalus Hookeri, Heterocycles, 2017, 94, 485-491.

18 W. X. Wang, J. S. Su, M. Yu, X. H. Liu, H. J. Yang, Z. M. Zou, Y. Zhang and C. Tang, Investigation on intestinal absorption ingredients and their absorption characteristics in Pterocephali Herba by everted intestinal sac method, China J. Chin. Mater. Med., 2019, 44, 3170-3177.

19 Y. J. Yu, L. Y. Zhu, W. Wang, X. Z. Huang, L. Gao, G. P. Li, Y. Yuan and Z. Y. Jiang, Studies on the chemical constituents of Tibetan medicine Pterocephalus hookeri. Proceedings of 2016 Academic Annual Meeting of Drug Clinical Evaluation Branch, Chinese Society of Ethnic Medicine, 2016, pp. 211-224.

20 C. Tang, H. J. Li, G. Fan, T. T. Kuang, X. L. Meng, Z. M. Zou and Y. Zhang, Network pharmacology and UPLC-Q-TOF/MS studies on the anti-arthritic mechanism of Pterocephalus hookeri, Trop. J. Pharm. Res., 2018, 17, 1095-1110.

21 Y. C. Wu, Y. J. Ying, F. J. Guo and G. F. Zhu, Bis-iridoid and lignans from traditional Tibetan herb Pterocephalus hookeri, Biochem. Syst. Ecol., 2014, 56, 209-212.

22 Y. C. Wu, Study on chemical constituents and pharmacological activities of Tibetan medicine P. hookeri, Shanghai University of Traditional Chinese Medicine, Shanghai, 2015.

23 Y. C. Wu, C. X. Guo, Y. Z. Zhu, Y. M. Li, F. J. Guo and G. F. Zhu, Four new bis-iridoids isolated from the traditional Tibetan herb Pterocephalus hookeri, Fitoterapia, 2014, 98, 104-109.

24 Y. C. Wu, J. Lu, X. Q. Y. Lu, R. Li, J. Guo, F. J. Guo and Y. M. Li, Monoterpenoids and Triterpenoids from Pterocephalus hookeri with NF-kB inhibitory activity, Phytochem. Lett., 2015, 13, 30-34.

25 Y. C. Wu, Y. J. Ying, F. J. Guo and G. F. Zhu, Secoiridoid/ iridoid subtype bis-iridoids from Pterocephalus hookeri, Magn. Reson. Chem., 2014, 52, 732-738.

26 G. Q. Li and D. L. Shen, Chemical constituents from Pterocephalus hookeri and their neuroprotection activities, Chin. Tradit. Pat. Med., 2018, 40, 1329-1335.

27 Y. L. Chen, H. Yu, F. J. Guo, Y. C. Wu and Y. M. Li, Antinociceptive and anti-inflammatory activities of a standardized extract of bis-iridoids from Pterocephalus hookeri, J. Ethnopharmacol., 2018, 216, 233-238.

28 C. X. Guo, Y. C. Wu, Y. Z. Zhu, C. Y. Wang, L. L. Tian, Y. Lu, C. Han and G. F. Zhu, In vitro and in vivo antitumor effects of
n-Butanol Extracts of Pterocephalus hookeri on Hep3B Cancer Cell, eCAM, 2015, 2015, 1-10.

29 J. X. Zhang, Y. X. Jing, J. W. Zhao, W. Wang, L. Gao, Z. Yang, J. K. Wang and Z. Y. Jiang, Studies on the chemical constituents of Tibetan medicine Pterocephalus hookeri, Her. Med., 2019, 38, 445-451.

30 W. X. Wang, S. Y. Luo, Y. Wang, X. Li, X. H. Liu, C. Tang and Y. Zhang, Pterocephanoside A, a new iridoid from a traditional Tibetan medicine, Pterocephalus hookeri, J. Asian Nat. Prod. Res., 2020, 1-8.

31 W. J. Li, Y. Gao, Y. L. Chen, Y. J. Wang and Y. Zhang, Simultaneous rapid determination of five chemical components in the Tibetan medicine Pterocephalus hookeri by UFLC-PDA. Modernization of Traditional Chinese Medicine and Materia Medica, World J. Sci. Technol., 2014, 1,34 .

32 J. F. Zhang, S. Huang, L. Z. Shan, L. Chen, Y. Zhang and X. L. Zhou, New Iridoid Glucoside from Pterocephalus hookeri, Chin. J. Org. Chem., 2015, 35, 2441-2444.

33 Z. Y. Dong, L. Wei, H. Q. Lu, Q. H. Zeng, F. C. Meng, G. W. Wang, X. Z. Lan, Z. H. Liao and M. Chen, Ptehoosines A and B: Two new sesamin-type sesquilignans with antiangiogenic activity from Pterocephalus hookeri (C.B. Clarke) Hoeck, Fitoterapia, 2021, 151, 104886.

34 Y. Zhang, W. J. Li, X. L. Meng, P. Shen and M. R. Jia, Studies on the chemicalconstituents of Tibetan medicine Pterocephalus hookeri, Journal of Chengdu University of Traditional Chinese Medicine, 2002, 25, 41-42.

35 R. Wang, L. Wei, Z. Y. Dong, F. C. Meng, G. Wang, S. Y. Zhou, X. Z. Lan, Z. H. Liao and M. Chen, Pterocephin A, a novel Triterpenoid Saponin from Pterocephalus hookeri induced liver injury by activation of necroptosis, Phytomedicine, 2021, 85, 153548.

36 X. M. Zhang, Study on the Chemical Constituents and Quality Evaluation Method for Pterocephalus hookeri, College of Chemistry and Chemical Engineering of Chongqing University, 2013.

37 X. J. Zhang, H. X. Su, C. W. He, Y. T. Wang, F. Q. Yang and J. B. Wan, Discrimination of Pterocephalus hookeri collected at flowering and non-flowering stages using GC MS-based fatty acid profiling, Anal. Methods, 2014, 6, 2141-2149.

38 B. Dinda, S. Debnath and Y. Harigaya, Naturally occurring iridoids. A review, part 1, Chem. Pharm. Bull., 2007, 55, 159-222.

39 B. Dinda, S. Debnath and Y. Harigaya, Naturally occurring secoiridoids and bioactivity of naturally occurring iridoids and secoiridoids. A review, part 2, Chem. Pharm. Bull, 2007, 55, 689-728.

40 B. Dinda, D. R. Chowdhury and B. C. Mohanta, Naturally occurring iridoids, secoiridoids and their bioactivity. An updated review, part 3, Chem. Pharm. Bull., 2009, 57, 765796.

41 A. Z. Wu, C. Z. Lin and C. C. Zhu, Progress in structureactivityrelationship of phenylethanoid glycosides, Nat. Prod. Res. Dev., 2013, 25, 862-865. 
42 L. L. Xiang, Y. J. Wen and M. S. Miao, Analysis of Chemical Pharmacological and Clinical Application of Eucommia Ulmoides Leaf, Acta Chin. Med., 2017, 32, 99-102.

43 H. Sun, J. Liu, A. Zhang, Z. Ying and X. Wang, Characterization of the multiple components of Acanthopanax senticosus stem by ultra HPLC with quadrupole time-of-flight tandem masss pectrometry, $J$. Sep. Sci., 2016, 39, 496-502.

44 C. Z. Wang, Z. Zhang, S. Anderson and C. S. Yuan, Natural products and chemotherapeutic agents on cancer: prevention vs. Treatment, Am. J. Chin. Med., 2014, 42, 1555-1558.

45 C. Zhao, J. H. Mao, J. M. Ai, W. M. Shen, T. L. Shi, D. Q. Zhang, X. N. Wang, Y. L. Wang and Y. P. Deng, Integrated lipidomics and transcriptomic analysis of peripheral blood reveals significantly enrichedpathways in type 2 diabetes mellitus, BMC Med. Genomics, 2013, 6, S12.

46 Y. H. Xiong, Y. Xu, L. Yang and Z. T. Wang, Gas chromatography-mass spectrometry-based profiling of serum fatty acids in acetaminophen-induced liver injured rats, J. Appl. Toxicol., 2012, 34, 149-157.

47 X. L. Wu, Y. D. Tong, K. Shankar, J. N. Baumgardner, J. Kang, J. Badeaux, T. M. Badger and M. J. J. Ronis, Lipid fatty acid profile analyses in liver and serum in rats with nonalcoholic steatohepatitis using improved gas chromatography-mass spectrometry methodology, J. Agric. Food Chem., 2011, 59, 747-754.

48 Y. J. Lee and Y. H. Kwon, Regulation of apoptosis and autophagy by luteolin in human hepatocellular cancer Hep3B cells, Biochem. Biophys. Res. Commun., 2019, 517, 617-622.

49 Y. Y. Wong, Y. Q. Tan, S. M. Lin and L. K. Leung, Apigenin and luteolin display differential hypocholesterolemic mechanisms in mice fed a high-fat diet, Biomed. Pharmacother., 2017, 1000.

50 S. J. Yang, P. J. Chen, S. H. Chang, Y. T. Weng, F. R. Chang, K. Y. Chang, C. Y. Chen, T. I. Kao and T. L. Hwang, Luteolin attenuates neutrophilic oxidative stress and inflammatory arthritis by inhibiting Raf1 activity, Biochem. Pharmacol., 2018, 154, 384-396.

51 X. L. Guan, Y. N. Yan, T. M. Wei, Z. H. Ren and C. S. Song, Experimental study on anti - inflammatory effect and acute toxicity of Pterocephalus hookeri, J. Tradit. Chin. Med., 2004, 27, 71-73.

52 L. Zhang, J. J. Hua, J. W. Lin, S. W. Fang and G. H. Du, Antiinflammatory and analgesic effects of ethanol and aqueous extracts of Pterocephalus hookeri (C.B. Clarke) Höeck, J. Ethnopharmacol., 2009, 123, 510-514.

53 X. F. Shen, Y. Zeng, J. C. Li, C. Tang, Y. Zhang and X. L. Meng, The anti-arthritic activity of total glycosides from Pterocephalus hookeri, a traditional Tibetan herbal medicine, Pharm. Biol., 2017, 55, 560-570.

54 I. Mcinnes and G. Schett, The pathogenesis of rheumatoid arthritis, N. Engl. J. Med., 2011, 365, 2205-2219.
55 Z. X. Yangben, D. Z. Pengmao, C. J. Xin, C. D. Zhuoma and B. Gong, Clinical Evaluation of Tibetan Medicine in Treating Rheumatoid Arthritis, J. Med. Pharm. Chin. Minorities, 2013, 5, 18-19.

56 J. Yang, F. Zuo, Z. C. Wei, Y. Zeng, X. L. Meng and Y. Zhang, Therapeutic Effects and Mechanisms of Total Glucosides of Herba Pterocephali on Adjuvant Arthritis Rats, Tradit. Chin. Drug Res. Clin. Pharmacol., 2016, 27, 788-794.

57 S. Ri and X. B. Zhou, Clinical observation of 101 cases of rheumatoid arthritis treated by Tibetan medicine, J. Med. Pharm. Chin. Minorities, 2017, 12, 36-37.

58 J. Wang, K. H. Zhao, Z. J. Geng, X. R. Lai, W. J. Yang, X. X. Hu, Y. Zhang and S. L. Jiangrong, Comparison Study on Zhenbu Disease of Tibetan Medicine and Bi Syndrome in Traditional Chinese Medicine, Modern Tradit Chin Med Mater Med, World J. Sci. Technol., 2015, 17, 2167-2171.

59 S. S. Song, L. L. Zhang and W. Wei, A review on animal model of experimental arthritis and its pathological mechanism, Chin. Pharmacol. Bull., 2011, 27, 1648-1653.

60 P. P. Li, G. X. Xie, S. S. Song, B. Huang, Y. J. Wu, Q. T. Wang, Y. Chang, Y. F. Li, A. W. Zhou, L. H. Liu, L. L. Zhang and W. Wei, Clinical manifestations and the main evaluation method on adjuvant-induced ar-thritis model in rats, Chin. J. Immunol., 2012, 28(05), 453-457.

61 X. D. Lei, G. F. Zhu, W. X. Cui, S. J. Fan, W. C. Zhong and X. Y. Jiang, Effect of total saponins from Pterocephalus hookeri on proliferation of tumor cells cultured in vivo, Lishizhen Med. Mater. Med. Res., 2011, 22, 1518-1519.

62 C. X. Guo, Y. C. Wu, Y. Z. Zhu, L. L. Tian, Y. H. C. Lu and G. F. Zhu, Inhibits Human Liver Hep3B Cell Proliferation and Invasion and Metastasis of n-butanol Part of Pterocephalus hookeri in Vitro, Chin. J. Exp. Tradit. Med. Formulae, 2015, 21, 100-105.

63 R. Wang, Z. Y. Dong, X. L. Zhang, J. X. Mao, F. C. Meng, X. Z. Lan, Z. H. Liao and M. Chen, Evaluation of the Liver Toxicity of Pterocephalus hookeri Extract via Triggering Necrosis, Toxins, 2019, 11, 142.

64 Y. J. Feng and Y. Zhang, Determination of oleanolic acid and ursolic acid in Tibetan medicine Pterycephalus by HPLCESLD method, Journal of Chengdu University of Traditional Chinese Medicine, 2007, 30, 54-56.

65 H. Quan, Z. J. Zheng, L. Q. Li, M. Chen and X. Z. Lan, Contents Determination of Oleanolic Acid and Ursolic Acid of Pterocephalus hookeri, Mod. Chin. Med., 2016, 18, 762765.

66 P. Yang, Y. Q. Li, X. Liu and S. X. Jiang, Determination of free isomeric oleanolic acid and ursolic acid in pterocephalus hookeri by capillary zone electrophoresis, J. Pharm. Biomed. Anal., 2007, 43, 1331-1334.

67 C. Tang, G. Fan, Q. Li, J. S. Su, X. L. Meng and Y. Zhang, Simultaneous determination of ten compounds in two main medicinal plant parts of Tibetan herb, Pterocephalus hookeri (CB Clarke) Höeck, by ultra-high performance liquid chromatography-photodiode array, Trop. J. Pharm. Res., 2017, 16, 1407-1416. 